\title{
Requirements with Regard to Nursing Consultation by mental Health Consultation Liaison Nurses and Suggestions for their Intervention
}

\author{
Mariko Kaneko ${ }^{1 *}$, Noriko Yamauchi ${ }^{2}$, Taeko Yasuda ${ }^{2}$, Hazuki Igita ${ }^{1}$ and Yoshiko Kawano \\ ${ }^{1}$ Tokyo Women's Medical University, School of Nursing, Graduate School of Nursing, Japan \\ ${ }^{2}$ Department of Nursing, Tokyo Women's Medical University, Japan
}

\begin{abstract}
Objective: This study aimed to analyze requirements with regard to nursing consultation by mental health consultation liaison nurses (MHCLNs) and examine suggestions for MHCLN intervention.

Methods: Subjects included 4 MHCLNs from a university hospital with approximately 1400 hospital beds located in the Tokyo metropolitan area and 143 patients and family members who either volunteered or were requested to participate by nurses. The consultation request route common to all 4 activity record formats, age and sex of the individual requesting counseling, and diagnosis and consultation data were retrospectively studied.

Results: Of the 143 subjects, 58 were male $(40.6 \%)$ and 85 were female $(59.4 \%)$, with a mean age of 54.2 years. Of 124 diagnoses, cancer was the most common ( $n=66,53.2 \%)$. With regard to patients' psychiatric status, acceptance of the disease was the most common difficulty $(n=39,27.3 \%)$, followed by adjustment disorder $(n=29$, $20.3 \%)$ and mood disorder $(n=10,7.0 \%)$. Psychiatric symptoms included anxiety $(n=75,45.1 \%)$, depression $(n=38$, $22.9 \%)$, and anger $(n=29,17.4 \%)$.

The consultation requests came most commonly from staff ( $n=66,46.2 \%$ ), followed by attending physicians, psychiatrists, or the palliative care team $(n=26,18.2 \%)$ and administrators $(n=26,18.3 \%)$. Twenty-two (15.7\%) requests were made directly by the patient or family member. MHCLNs collaborated with psychiatrists for 74 (51.7\%) subjects, whereas they did not collaborate for 68 subjects $(47.6 \%)$.

Details of direct care included interviews using supportive psychotherapy $(n=92,64.3 \%)$ combined with relaxation $(n=18,12.5 \%)$ and an approach to partial recognition $(n=11,7.7 \%)$. Nursing consultations totaled $127(88.8 \%)$.

Conclusions: Among the subjects consulted by an MHCLN, those that were counseled by a nurse alone (i.e., no liaison with the psychiatry department) often had difficulty in accepting the disease or were maladjusted with symptoms such as anxiety, depression, or anger. If these symptoms can be relieved during the early stages, medication may not be required and consultation or practice may prevent deterioration in the psychiatric condition and lead to an improvement in symptoms. In patients with somatic disorders requiring psychological care, nurses highly specialized in mental health care, such as MHCLNs, are required to serve as liaison personnel. This is especially required for patients with psychological problems caused by the stress of the disease and treatment, albeit within normal limits, or for patients in a condition requiring psychiatric treatment.
\end{abstract}

Keywords: Consultation; Mental health consultation liaison nurses; Certified nurse specialists mental health nursing; Nursing role

\section{Introduction}

Although the mental health care of patients with physical illness is a high priority in medical care, the roles and duties of mental health nurses remain unclear [1]. The usefulness of interventions by mental health consultation liaison Nurses (MHCLNs) for patients with physical illness has been demonstrated [1-3]. With regard to psychiatric or psychological problems of patients with somatic disorders, it is important for MHCLNs to coordinate with the medical care team and support the patient's quality of life [2-4].

As of December 2012, the number of qualified mental health certified nurse specialists (CNSs) registered in the Japanese Nursing Association is insufficient ( $n=116),[5]$ and at this point in time, the consultation needs remain unclear. In this study, CNS refers to an internationally recognized clinical nurse specialist.

This study aimed to analyze requirements with regard to nursing consultation by MHCLNs and examine suggestions for MHCLN intervention.

\section{Participants and Methods}

\section{Design}

A retrospective study was conducted.

\section{Survey period}

The survey period was from April 2011 to March 2012.

\section{Method of data collection}

In this study, an MHCLN was defined as an individual who had completed a postgraduate Masters course in psychiatric mental health. MHCLNs serve 6 functions in accordance with the Japanese Nursing Association guidelines for CNS: practice, consultation, coordination, ethical coordination, education, and research.

The subjects included 4 MHCLNs from a university hospital located in the Tokyo metropolitan area, which has approximately 1400 hospital

*Corresponding author: Mariko Kaneko, Tokyo Women's Medical University School of Nursing, Graduate School of Nursing, 8-1 Kawada-cho, Shinjuku-ku, Tokyo 162-8666, Japan, Tel: +81-3-3353-8111; Fax: +81-3341-8832; E-mail: kaneko.mariko@twmu.ac.jp

Received January 24, 2013; Accepted April 30, 2013; Published May 06, 2013

Citation: Kaneko M, Yamauchi N, Yasuda T, Igita H, Kawano Y (2013) Requirements with Regard to Nursing Consultation by mental Health Consultation Liaison Nurses and Suggestions for their Intervention. J Nurs Care S5: 007. doi: 10.4172/2167-1168.S5-007

Copyright: $\odot 2013$ Kaneko M, et al. This is an open-access article distributed under the terms of the Creative Commons Attribution License, which permits unrestricted use, distribution, and reproduction in any medium, provided the original author and source are credited. 
Citation: Kaneko M, Yamauchi N, Yasuda T, Igita H, Kawano Y (2013) Requirements with Regard to Nursing Consultation by mental Health Consultation Liaison Nurses and Suggestions for their Intervention. J Nurs Care S5: 007. doi: 10.4172/2167-1168.S5-007

Page 2 of 3

beds, and 143 patients and family members who either volunteered or were requested to participate by nurses. The consultation request route from activity record sheets pertaining to all CNSs, age and sex of the individual requesting counseling, and diagnosis as well as consultation and intervention data were retrospectively studied. Of the 4 MHCLNs, 3 had completed a postgraduate Masters course in psychiatric mental health and 1 had completed a doctorate.

Two were MHCLNs working under contract at the university hospital. The remaining 2 were faculty at the University school of Nursing, and they also worked as MHCLNs in the university hospital.

\section{Ethical considerations}

Personal data were kept confidential, and written consent was obtained from the affiliated Department of Nursing. The study was approved in August 2012 by the Ethics Committee of Tokyo Women's Medical University (No: 2356).

\section{Results}

Of the 143 participants, 58 were male $(40.6 \%)$ and 85 were female (59.4\%), with a mean age of54.2 years (range, $0-88$ years). There were 115 patients $(80.4 \%)$ and 28 family members (19.6\%).Twenty-five subjects $(17.5 \%)$ had a history of mental illness while118 (82.5\%) had no such history.

A total of 19 patients could not be diagnosed after detailed examination. Of 124 diagnoses, cancer was the most common $(n=66$, $53.2 \%)$, and $25(37.8 \%)$ of these patients required treatment in coordination with the palliative care team. Other illnesses included cardiac disease $(n=9,7.3 \%)$, endocrine disease $(n=9,7.3 \%)$, gastrointestinal disease $(n=7,5.6 \%)$, intracranial disease $(n=5,4.0 \%)$, psychiatric illness

\begin{tabular}{|c|c|}
\hline Diagnosis & Number of cases (\%) \\
\hline Cancer & $66(53.2)$ \\
\hline Cardiac disease & $9(7.3)$ \\
\hline Endocrine disease & $9(7.3)$ \\
\hline Gastrointestinal disease & $7(5.3)$ \\
\hline Intracranial disease & $5(4.0)$ \\
\hline Psychiatric illness & $5(4.0)$ \\
\hline Dermatological disease & $3(2.5)$ \\
\hline Neurological disease & $2(1.6)$ \\
\hline Other & $18(14.5)$ \\
\hline Total & 124 \\
\hline
\end{tabular}

Psychological symptoms included anxiety $(n=75,45.1 \%)$, depression ( $n=38$, $22.9 \%)$, and anger ( $n=29,17.4 \%$; Figure 1$)$.

Table 1: Diagnoses and number of subjects who were consulted by nurses.

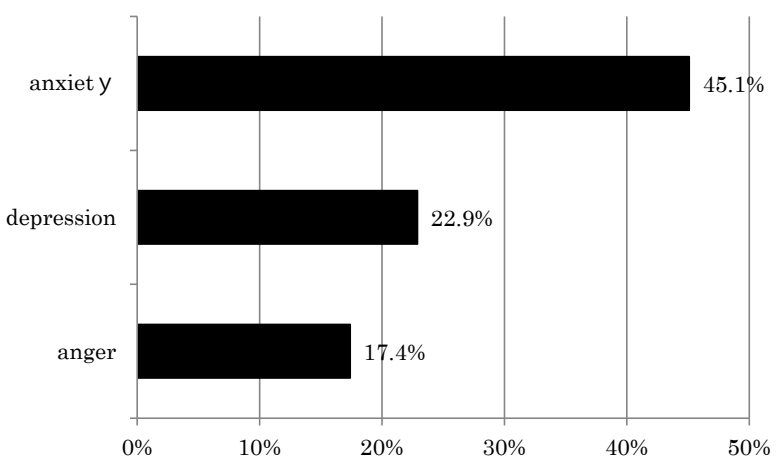

Figure 1: Incidence of psychological symptoms.

\begin{tabular}{|c|c|}
\hline Mental state & Number of cases (\%) \\
\hline Difficulty accepting the disease & $39(27.3)$ \\
\hline Adjustment disorder & $29(20.3)$ \\
\hline Delirium & $14(9.8)$ \\
\hline Mood disorder & $10(7.0)$ \\
\hline Anxiety disorder & $8(5.6)$ \\
\hline Eating disorder & $3(2.1)$ \\
\hline Substance-related disorder & $2(1.4)$ \\
\hline Schizophrenia & $2(1.4)$ \\
\hline Personality disorder & $1(0.7)$ \\
\hline Developmental disorder & $1(0.7)$ \\
\hline Other & $6(14.2)$ \\
\hline Unknown & $27(8.4)$ \\
\hline Total & $143(100)$ \\
\hline
\end{tabular}

Table 2: Mental state of subjects.

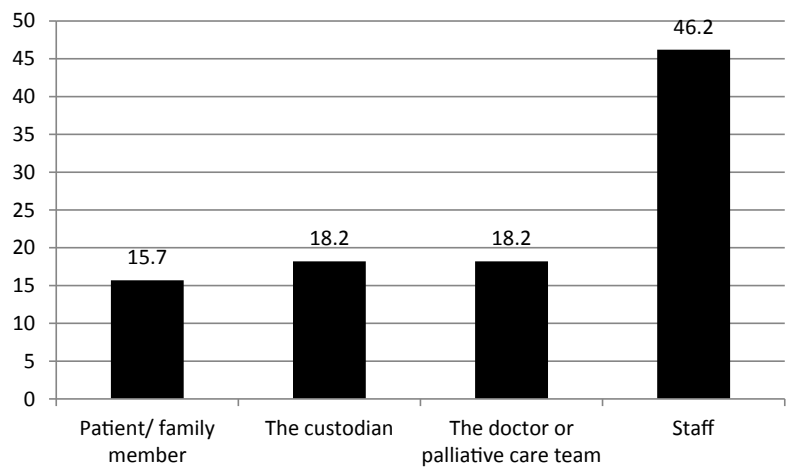

Figure 2: Route of requesting consultation by mental health consultation liaison nurses.

In this study, the palliative care team comprised doctors specializing in palliative care, a clinical nurse specialist (CNS) for cancer patients, a pharmacist, a psychiatrist, and a psychologist.

( $n=5,4.0 \%)$, dermatological disease $(n=3,2.5 \%)$, neurological disease $(n=2,1.6 \%)$, and others $(n=18,14.5 \%$; Table 1 ; Figure 1$)$.

The most common mental status was difficulty in accepting the disease $(n=39,27.3 \%)$, followed by adjustment disorder $(n=29,20.3 \%$; Table 2). These data includes subjects who had not consulted the psychiatric department.

The consultation requests came most commonly from staff $(n=66$, $46.2 \%)$, followed by attending physicians, psychiatrists, or the palliative care team $(n=26,18.2 \%)$ and administrators $(n=26,18.3 \%)$. Twenty-two $(15.7 \%)$ requests were made directly by the patient or family member (Figure 2). MHCLNs collaborated with psychiatrists for 74 (51.7\%) subjects, whereas they did not collaborate for 68subjects (47.6\%).

The number of MHCLN interventions per case varied from 1 to 13, with a mean number of 3 interventions (31.5\%) being the most common. Direct care included interviews using supportive psychotherapy $(n=92$, $64.3 \%)$, combined with relaxation $(n=18,12.5 \%)$ and an approach to partial recognition $(n=11,7.7 \%)$.Nursing consultations totaled 127 (88.8\%). Coordination with other occupations, including doctors, was required for 110 subjects $(76.9 \%)$. Ethical adjustments were required for 16 subjects $(11.8 \%)$.

\section{Discussion}

The main reason for consultations being most commonly requested for cancer patients is possibly because the institutions dealt with were 
Citation: Kaneko M, Yamauchi N, Yasuda T, Igita H, Kawano Y (2013) Requirements with Regard to Nursing Consultation by mental Health Consultation Liaison Nurses and Suggestions for their Intervention. J Nurs Care S5: 007. doi: 10.4172/2167-1168.S5-007

Page 3 of 3

characteristically designated as cancer care hospitals. The incidence of depression and adjustment disorder is high in cancer patients; furthermore, there is a great need for psychological and mental health care [6-8]. However, medical staff in the field of oncology have pointed out that cancer, depression, and delirium are insufficiently screened $[9,10]$. It was revealed that there is a wide spread requirement for consultations with MHCLNs. According to a survey of 88 oncologyrelated CNSs in Japan, more than 50\% nurses engaged in cancerrelated nursing care were very concerned about assessing anxiety and depression in cancer patients and approximately $20 \%$ were extremely concerned about caring for patients experiencing depression and anger [11].

In the present study, among the subjects consulted by an MHCLN, those that were consulted by an MHCLN alone without liaison with the psychiatry department often had difficulty in accepting the disease or showed symptoms of adjustment disorder such as anxiety, depression, and anger. If these symptoms are relieved during the early stage, medication may not be required and consultation or practice may prevent deterioration of the mental condition and lead to improvement in symptoms. In addition, in the field of cancer-related nursing, nurses are the key providers of physical and psychiatric care and can be trained to deliver multifaceted interventions for psychosocial problems [12-15]. According to the results of this study, nurses who are highly specialized in mental health care, such as MHCLNs, are required to act as liaison personnel in order to provide better care for patients with somatic disorders, which require specific psychological care. This is especially required for patients with psychological problems caused by the stress of the disease and treatment, albeit within normal limits, or for patients in a condition requiring psychiatric treatment.

Our findings suggest that in order to ensure early interventions in patients requiring psychological care, nurses should work closely with both physicians and the mental health team when required, thereby providing appropriate and timely nursing care for patients with physical illnesses and in need of psychiatric care. This should take place from the time the request is made to the time when treatment is administered.

\section{Limitations and Future Research}

The sample in the study was too small for the findings to be generalized or extrapolated.

In future, we need to create a system wherein nursing staff, CNSs for cancer patients, and MHCLNs cooperate with patients to correctly assess the patient both physically and mentally. This will result in patients receiving appropriate and timely physical and mental care. In addition, we should examine methods of providing holistic care in university hospitals that provide a high standard of care.

\section{Conclusions}

In this study, cancer patients accounted for more than half the patients with nursing requirements concerning their psychiatric health. The most common mental conditions were difficulty in accepting the illness, followed by adjustment. These findings suggest that early intervention is required for such patients. Therefore, specialist mental health care nurses should coordinate with general nurses and psychiatrists and be a part of the medical team.

\section{Acknowledgement}

A part of this study was presented at the 8th Tokyo Women's Medical University Nursing Society in October 2012. This work was supported by a Grant-in-Aid for Scientific Research, Number 22592467

\section{References}

1. Rylance R, Chapman H, Harrison J (2012) Who assesses the physical health of inpatients? Mental Health Practice 16: 14-20.

2. Dorogi Y, Campiotti C, Gebhard S (2012) Liaison psychiatry nurse: The development of supervision in somatic medicine. Encephale.

3. Bossis ML, Lemoine NC, Guitteny M, Vanelle JM, Sauvaget A, et al. (2012) The psychiatric liaison nurse in somatic care departments. Soins: 16-18.

4. Silva NG, de Oliveira AG, Ide PH (2011) Demands of psychiatric care in a university hospital. Rev Gaucha Enferm 32: 531-538.

5. Japanese Nursing Association, 2012.

6. Fallowfield L, Ratcliffe D, Jenkins V, Saul J (2001) Psychiatric morbidity and its recognition by doctors in patients with cancer. Br J Cancer 84: 1011-1015.

7. Bringmann H, Singer S, Höckel M, Stolzenburg JU, Krauss O, et al. (2008) Longitudinal analysis of psychiatric morbidity in cancer patients. Onkologie 31 343-344.

8. Akechi T, Okuyama T, Sugawara Y, Nakano T, Shima Y, et al. (2004) Major depression, adjustment disorders, and post-traumatic stress disorder in terminally ill cancer patients: associated and predictive factors. J Clin Onco 22: $1957-1965$

9. McDonald MV, Passik SD, Dugan W, Rosenfeld B, Theobald DE, et al. (1999) Nurses' recognition of depression in their patients with cancer. Oncol Nurs Forum 26: 593-599.

10. Keller M, Sommerfeldt S, Fischer C, Knight L, Riesbeck M, et al. (2004) Recognition of distress and psychiatric morbidity in cancer patients: a multimethod approach. Ann Oncol 15: 1243-1249.

11. Kaneko M, Ryu S, Nishida H, Tamasato K, Shimodaira Y, et al. (2012) Nurses' recognition of the mental state of cancer patients and their own stress management - a study of Japanese cancer-care nurses. Psychooncology.

12. Hellbom M, Brandberg Y, Kurland J, Arving C, Thalén-Lindström A, et al. (2001) Assessment and treatment of psychosocial problems in cancer patients: an exploratory study of a course for nurses. Patient Educ Couns 45: 101-106.

13. Strong V, Sharpe M, Cull A, Maguire P, House A, et al. (2004) Can oncology nurses treat depression? A pilot project. J Adv Nurs 46: 542-548.

14. Strong V, Waters R, Hibberd C, Murray G, Wall L, et al. (2008) Management of depression for people with cancer (SMaRT oncology 1): a randomised trial. Lancet 372: 40-48.

15. Fukui S, Ogawa K, Ohtsuka M, Fukui N (2009) Effect of communication skills training on nurses' detection of patients' distress and related factors after cancer diagnosis: a randomized study. Psychooncology 18: 1156-1164. 